\title{
Temporal and spatial association of histone H2A variant hv1 with transcriptionally competent chromatin during nuclear development in Tetrahymena thermophila
}

\author{
Laurie A. Stargell,, Josephine Bowen, ${ }^{2}$ Christopher A. Dadd, ${ }^{3}$ Peter C. Dedon, ${ }^{4}$ Maria Davis, ${ }^{5}$ \\ Richard G. Cook, ${ }^{6}$ C. David Allis, ${ }^{3}$ and Martin A. Gorovsky ${ }^{2,7}$ \\ ${ }^{1}$ Department of Biological Chemistry and Molecular Pharmacology, Harvard Medical School, Boston, Massachusetts \\ 02115 USA; ${ }^{2}$ Biology Department, University of Rochester, Rochester, New York 14627-0214 USA; ${ }^{3}$ Department of Biology, \\ Syracuse University, Syracuse, New York 13244-1270 USA; ${ }^{4}$ Division of Toxicology, 16-336B, Whitaker College of Health \\ Sciences \& Technology, Massachusetts Institute of Technology, Cambridge, Massachusetts 02139 USA; ${ }^{5}$ United States \\ Biochemical Corp., Cleveland, Ohio 44128 USA; ${ }^{6}$ Department of Microbiology and Immunology, Baylor College of \\ Medicine, Houston, Texas 77030 USA
}

\begin{abstract}
Vegetative cells of the ciliated protozoan Tetrahymena thermophila contain a transcriptionally active macronucleus and a transcriptionally inactive micronucleus. Although structurally and functionally dissimilar, these nuclei are products of a single postzygotic division during conjugation, the sexual phase of the life cycle. Immunocytochemical analyses during growth, starvation, and conjugation were used to examine the nuclear deposition of hv1, a histone H2A variant that is found in macronuclei and thought to play a role in transcriptionally active chromatin. Polyclonal antisera were generated using whole hv1 protein and synthetic peptides from the amino and carboxyl domains of hv1. The transcriptionally active macronuclei stained at all stages of the life cycle. Micronuclei did not stain during growth or starvation but stained with two of the sera during early stages of conjugation, preceding the stage when micronuclei become transcriptionally active. Immunoblot analyses of fractionated macro- and micronuclei confirmed the micronuclear acquisition of hv1 early in conjugation. hv1 staining disappeared from developing micronuclei late in conjugation. Interestingly, the carboxy-peptide antiserum stained micronuclei only briefly, late in development. The detection of the previously sequestered carboxyl terminus of hv1 may be related to the elimination of hv1 during the dynamic restructuring of micronuclear chromatin that occurs as the micronucleus enters a transcriptionally incompetent state that is maintained during vegetative growth. These studies demonstrate that the transcriptional differences between macro- and micronuclei are associated with the loss of a chromatin component from developing micronuclei rather than its de novo appearance in developing macronuclei and argue that hv1 functions in establishing a transcriptionally competent state of chromatin.
\end{abstract}

[Key Words: Histone H2A; T. thermophila; macronuclei; chromatin; transcription]

Received August 4, 1993; revised version accepted October 20, 1993.

Meiosis and gamete formation, followed by fertilization, determination, and differentiation are programmed developmental events in higher eukaryotes, where individual gametes unite and the zygote proceeds through embryogenesis to produce morphologically and functionally distinct tissues. These developmental events also occur during sexual reproduction in the unicellular ciliate, Tetrahymena thermophila. However, as opposed to the

\footnotetext{
${ }^{7}$ Corresponding author.
}

morphologically and functionally distinct tissues generated at the endpoint of sexual reproduction in higher eukaryotes, conjugation in Tetrahymena generates morphologically and functionally distinct nuclei that reside in a single cell.

Tetrahymena, like all ciliates, have two types of nuclei: a diploid micronucleus that serves as the germ line of the cell, and a polyploid somatic macronucleus that controls the phenotype of the cell and is destroyed during the sexual process of conjugation (Gorovsky 1973, 
1980). During vegetative growth, the micronucleus divides mitotically and is transcriptionally inert while the macronucleus divides amitotically and is transcriptionally active. Differences in chromatin composition between the two types of nuclei are associated with their functional dichotomy. Although both nuclei possess the four core histones $(\mathrm{H} 2 \mathrm{~A}, \mathrm{H} 2 \mathrm{~B}, \mathrm{H} 3$, and $\mathrm{H} 4$; for review, see Gorovsky 1986), these proteins are highly acetylated in macronuclei while histone acetylation is greatly reduced in micronuclei (Vavra et al. 1982; Chicoine et al. 1987). In addition, the macro- and micronuclei contain dramatically different linker proteins. Macronuclei have an H1-like protein that has many properties characteristic of $\mathrm{Hl}$ in higher organisms (Gorovsky et al. 1974). H1 is lacking in micronuclei (Gorovsky et al. 1975; Johmann and Gorovsky 1976). Instead, micronuclei contain a set of unique linker-associated polypeptides $(\alpha, \beta, \gamma$, and $\delta)$ that lack typical Hl-like properties (Allis et al. 1984).

Macronuclei also contain two minor histone variants, hv1 (an $\mathrm{H} 2 \mathrm{~A}$ variant) and hv2 (an $\mathrm{H} 3$ variant), which are absent from micronuclei of vegetative cells (Allis et al. $1980 \mathrm{~b})$. Antibodies specific to hvl cross-react on Western blots with acid-soluble nuclear proteins from animal (mouse), plant (wheat), and fungal (Saccharomyces cerevisiae) kingdoms (Allis et al. 1986). Sequence analysis (Allis et al. 1986; White et al. 1988; Van Daal et al. 1990) indicates that the central core region of hvl is strikingly similar to H2A.F of chicken (Harvey and Whiting 1983; Dalton et al. 1989), H2A.Z of mammals (Hatch and Bonner 1988, 1990), and H2A.F/Z of sea urchins (Ernst et al. 1987). An hv1 cDNA clone was used to clone H2AvD, an H2A variant of Drosophila melanogaster (Van Daal et al. 1988, 1990), which is encoded by an essential, singlecopy gene (Van Daal and Elgin 1992). Comparison of the derived protein sequences of the major and variant $\mathrm{H} 2 \mathrm{As}$ led to the surprising observations that the variants in different organisms are more similar to each other than each is to the major H2A in the same organism (White et al. 1988; Van Daal et al. 1990), and that the variant H2As actually show less evolutionary divergence than the major H2As. These results indicate that there were two types of $\mathrm{H} 2 \mathrm{~A}$ genes in primitive eukaryotes before the divergence of the major eukaryotic groups and that the major and variant $\mathrm{H} 2 \mathrm{As}$ have been under different selective pressures since that time. Therefore, the major and variant $\mathrm{H} 2 \mathrm{As}$ must have distinct and important functions.

Analysis of histone proteins in a variety of organisms has identified a variant for every major histone type ( $\mathrm{Wu}$ and Bonner 1981; Wu et al. 1982; Zweidler 1984; Schümperli 1986; Wu et al. 1988; Sadler and Brunk 1992). The existence of histone variants with synthesis that is temporally or developmentally regulated suggests the possibility that nucleosomes containing different variants may perform distinct functions. Coupled with the fact that nucleosomes are general repressors of transcription (for review, see McGhee and Felsenfeld 1980; Grunstein 1990) and that mutations in $\mathrm{H} 4$ can affect repression and activation of specific genes (Park and Szostak 1990), one possible function for variant nucleo- somes may be a role in the regulation of transcriptional activity of specific genes.

There is circumstantial evidence that hvl plays a role in the establishment of a transcriptionally competent state of chromatin. Immunofluorescence studies using an hvl-specific antiserum demonstrated that determinants recognized by these antibodies are highly concentrated in small dots in mammalian nucleoli, presumably in the chromatin containing highly active ribosomal genes (Allis et al. 1982). hvl determinants are also associated with a subset of loci in Drosophila polytene chromosomes that are, were, or will be active during the third larval instar or prepupal stage of development (S. Elgin, pers. comm.). Coupled with the specific localization of hvl in transcriptionally active macronuclei of vegetative Tetrahymena, these studies suggest that hvl and proteins antigenically related to it in mammalian cells and Drosophila are preferentially associated with transcriptionally active or potentially active (transcriptionally competent/ chromatin.

It has been shown that micronuclei are transcriptionally active for a brief period of time, during meiotic prophase in early conjugation (Sugai and Hiwatashi 1974; Martindale et al. 1985). Recent observations show that TATA-binding protein (TBP), a component necessary for transcription by all three nuclear RNA polymerases (for review, see White and Jackson 1992; Rigby 1993), is acquired by micronuclei just before this period of transcriptional activity (Stargell and Gorovsky 1993). Anti-TBP antibodies stain micronuclei just before and continue to stain micronuclei after this period of transcriptional activity, whereas micronuclei in starved and growing cells do not stain. Previous immunocytochemical studies using anti-hvl antibodies generated against gel-purified whole hvl protein (anti-hvl-gel serum) demonstrated a macronuclear-specific localization of hvl during growth and starvation; hvl was also found to accumulate in developing macronuclei late during conjugation, approximately coincident with the acquisition of transcriptional activity in these nuclei (Wenkert and Allis 1984). However, hvl staining during early stages of conjugation was not detected in these initial studies. Therefore, transcription in prophase micronuclei appeared to occur in the absence of hvl, casting doubt on the association of hvl with transcriptionally competent chromatin.

We wished to reexamine the nuclear distribution of hvl using recently obtained hvl antisera of higher titer and the paraformaldehyde fixation technique used in the anti-TBP studies. Three polyclonal antisera were generated against different hvl immunogens: HPLC-purified whole hvl protein, an amino-proximal peptide, and a carboxy-terminal peptide. Mature macronuclei stain with all three antisera during most stages of the life cycle. Loss of mature macronuclear staining occurs coincident with the degradation and eventual elimination of the parental macronucleus during late conjugation. Thus, macronuclear staining is consistent with postulated association of hvl with transcriptionally competent chromatin. The transcriptionally inert micronuclei 
did not stain during growth or starvation; however, micronuclei did stain with anti-hvl-HPLC and anti-amino sera during conjugation. The onset of micronuclear staining precedes the brief window of transcriptional activity observed during meiotic prophase (Sugai and $\mathrm{Hi}$ watashi 1974; Martindale et al. 1985), consistent with the hypothesis that hvl acquisition is important for transcriptional potential. Biochemical purification of both macronuclei and micronuclei, followed by immunoblot analyses confirmed the developmentally regulated acquisition of hvl during early conjugation. Surprisingly, the immunocytochemical staining of hvl persisted even after micronuclei stopped synthesizing RNA and only disappeared from developing micronuclei late in conjugation, coincident with the onset of transcription in developing macronuclei and the degeneration of the parental macronucleus. In contrast, anti-carboxy antibodies stained micronuclei only briefly, late in development, just before loss of detection of hvl by all three sera. We suggest that the carboxyl terminus of this histone is sequestered in micronuclei early in conjugation and that its detection in later stages may be attributable to a change in conformation or to loss of associated proteins preceding its degradation. These results argue strongly that hvl is associated with transcriptional competence and demonstrate that micronuclear chromatin is actively restructured late in development to eliminate proteins specific to macronuclei in vegetative cells.

\section{Results}

\section{Generation and characterization of hv1 antisera}

Three polyclonal antisera were generated to distinct immunogens of the hvl protein (Fig. la). HPLC-purified hvl protein was used to generate an antiserum against the whole protein (anti-hvl-HPLC). Anti-amino serum was produced by immunization with a peptide corresponding to amino acids 17-28, whereas anti-carboxy serum was generated against a peptide corresponding to amino acids 129-145 (White et al. 1988). The specificity of each antibody was investigated by immunoblot assays against histone preparations separated by SDS-PAGE. Under the conditions described, all three immune antisera reacted solely with the hvl band (Fig. lb-d). When histone preparations were separated by triton/acid/ urea-PAGE (Allis et al. 1979; Allis et al. 1980a), which alters the relative mobility of the histones, staining was again specific to the hvl region (data not shown). These immunoblot results indicate all three sera are highly specific to the minor histone variant, hvl.

Competition experiments using hvl synthetic peptides in immunoblot assays were performed to determine whether the three sera recognize distinct antigens of the hvl protein. Detection of hvl with anti-hvlHPLC serum is not competed with amino-proximal peptide. Carboxy-terminal peptide depletes the staining significantly but does not remove it (Fig. 1b). Competition with a peptide corresponding to the variant box (amino acids 48-59) also diminishes the staining slightly. A

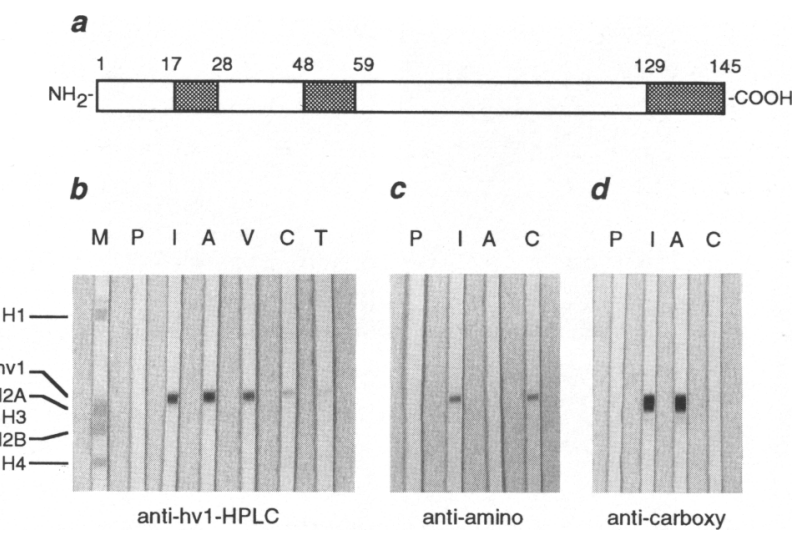

Figure 1. Characterization of hvl antisera and competition with peptides in immunostaining. (a) Diagram of hvl protein. Relative amino acid positions of the synthetic peptides are depicted by shaded boxes. $(b-d)$ Tetrahymena histones were separated on a SDS- $15 \%$ polyacrylamide gel and electroblotted to nitrocellulose, which was cut into strips. In some cases, as listed below, immune sera were preincubated with a 100-fold molar excess of peptide. Serum reaction mixes applied to the strips were preimmune $(\mathrm{P})$; immune $(\mathrm{I})$; immune plus amino peptide (A); immune plus variant box peptide (V); immune plus carboxy peptide $(C)$; immune plus all three peptides $(T)$. Antisera used: anti-hvl-HPLC $(b)$; anti-amino $(c)$; anti-carboxy $(d)$. The positions of the histones are indicated on the Ponceau S-stained strip (M). All three immune sera (I) stain only hvl; preimmune sera $(\mathrm{P})$ do not stain any histone bands.

mixture of all three peptides does not totally abolish anti-hvl-HPLC reactivity with hvl protein, suggesting that although some of the antibodies in this polyclonal serum recognize the carboxyl terminus and variant box of hvl, others must detect different epitopes. In contrast, anti-amino and anti-carboxy antibodies can be competed completely by their respective peptides, with no effect by the alternate peptide (Fig. lc,d).

\section{Immunocytochemical staining of growing and starved cells with anti-hv1-HPLC is macronuclear specific}

The subcellular localization of hvl was determined by fluorescence microscopy of growing or starved cells after fixation, incubation with anti-hv1-HPLC antibodies, and detection with fluorescein-labeled secondary antibodies. Staining with anti-hvl-HPLC was found to be localized solely to macronuclei in both growing and starved cells (Fig. 2).

\section{Anti-hv1-HPLC serum also stains micronuclei in early conjugation}

During conjugation, the sexual stage of the life cycle, micronuclei undergo an extended period of meiosis, followed by nuclear exchange, fertilization, postzygotic divisions, and differentiation to eventually produce new macro- and micronuclei. At the end of this process, the parental macronucleus in each cell is degraded. As ex- 

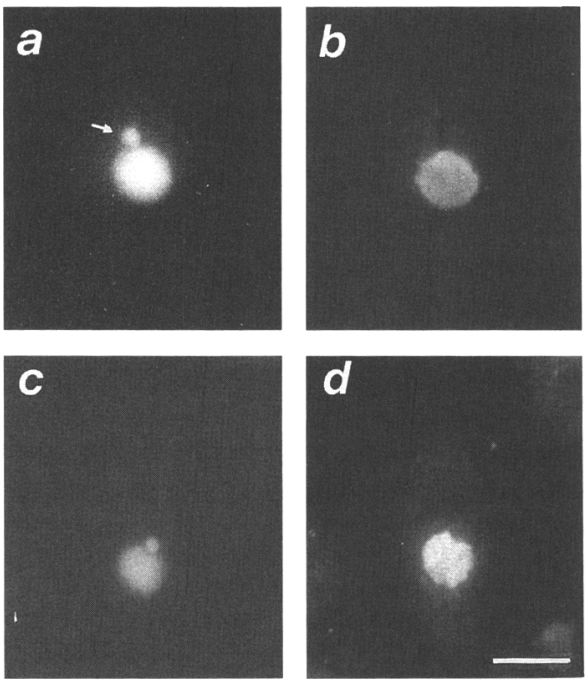

Figure 2. Macronuclear-specific localization of hvl in growing and starved Tetrahymena. Immunofluorescent images of a growing cell stained with DAPI $(a)$ and with anti-hvl-HPLC $(b)$. A starved cell stained with DAPI $(c)$ and anti-hvl-HPLC $(d)$. The arrow indicates the micronucleus. Bar, $10 \mu \mathrm{m}$.

pected, anti-hv1-HPLC stains the parental macronucleus throughout development until these nuclei cease transcriptional activity and begin to degenerate (Figs. 3 and 4). Surprisingly, this serum also stains micronuclei during both meiotic and gametic stages (Fig. 3). This staining appears in early meiosis $(\sim 1.5 \mathrm{hr}$ after mixing cells of opposite mating types) and persists through crescent stage and gametogenesis. The onset of micronuclear staining with anti-hvl-HPLC precedes the onset of transcriptional activity in these nuclei, which is observable after the micronuclei begin to elongate (the stage shown in Fig. 3c; Sugai and Hiwatashi 1974; Martindale et al. 1985). Detectable levels of transcription cease by the time the nuclei are fully elongated and crescent shaped (the stage shown in Fig. 3e). However, hvl staining persists long after micronuclear transcription ceases. Micronuclear staining is detectable in the products of the second postzygotic division, even those nuclei positioned in the posterior of the cell, which are destined to develop into mature micronuclei (Fig. 4b). Staining with antihvl-HPLC is lost from micronuclei and becomes specific to developing macronuclei coincident with the onset of transcription from the new macronuclei and the degradation of the parental macronucleus (Fig. 4f). The loss of hvl staining in developing micronuclei occurs after they have assumed a position in the center of the cell, in close proximity to the still positive macronuclei.

Pre-immune serum does not stain any subcellular structures in Tetrahymena cells (data not shown). Furthermore, staining of all nuclei (parental macronuclei, developing macronuclei, and micronuclei) with an antibody to the major $\mathrm{H} 2 \mathrm{~A}$ is easily detectable throughout conjugation, even at late stages, indicating that micronuclear chromatin is accessible to anti-histone antibodies (data not shown).

\section{Nuclear fractionation and immunoblotting demonstrates the acquisition of hv1 by micronuclei during early conjugation}

The unexpected finding that micronuclei stain with anti-hv1-HPLC during early conjugation prompted the biochemical purification of these nuclei to confirm the sudden developmentally regulated acquisition of hvl. Nuclei were harvested from cells in log phase growth, starvation, or $2.5 \mathrm{hr}$ after the initiation of conjugation, formaldehyde fixed, and subjected to sedimentation at
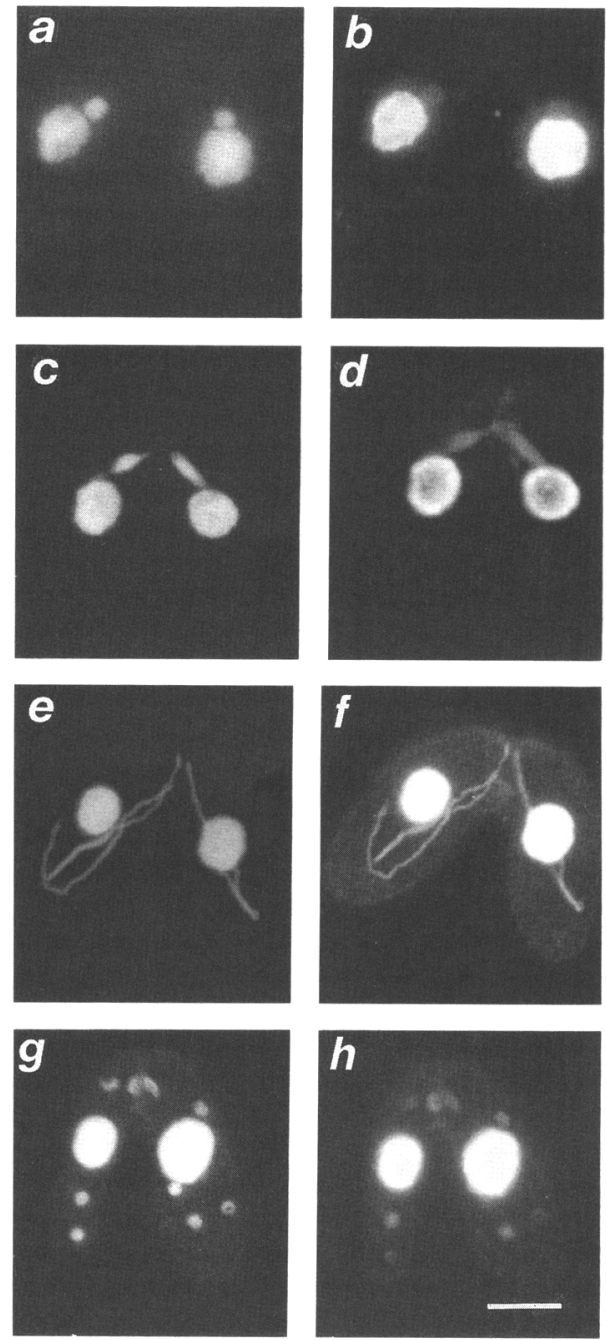

Figure 3. During conjugation both macronuclei and micronuclei are stained with anti-hvl-HPLC serum. Mating cells were visualized with DAPI stain $(a, c, e, g)$ and with anti-hv1-HPLC serum $(b, d, f, h)$. Mating pairs from early conjugation: The micronucleus has just moved away from the macronucleus, at $\sim 1.5 \mathrm{hr}$ after mixing $(a, b)$; meiotic prophase, stage II, at $2.5 \mathrm{hr}$ $(c, d)$; crescent stage of meiotic prophase, at $3.5 \mathrm{hr}(e, f)$; third prezygotic division, $\sim 5 \mathrm{hr}$ after mixing $(g, h)$. Times given are approximate and are the earliest point after mixing cells when the stages can generally be observed (for a complete description of the conjugation process in Tetrahymena, see Martindale et al. 1982). Bar, $10 \mu \mathrm{m}$. 

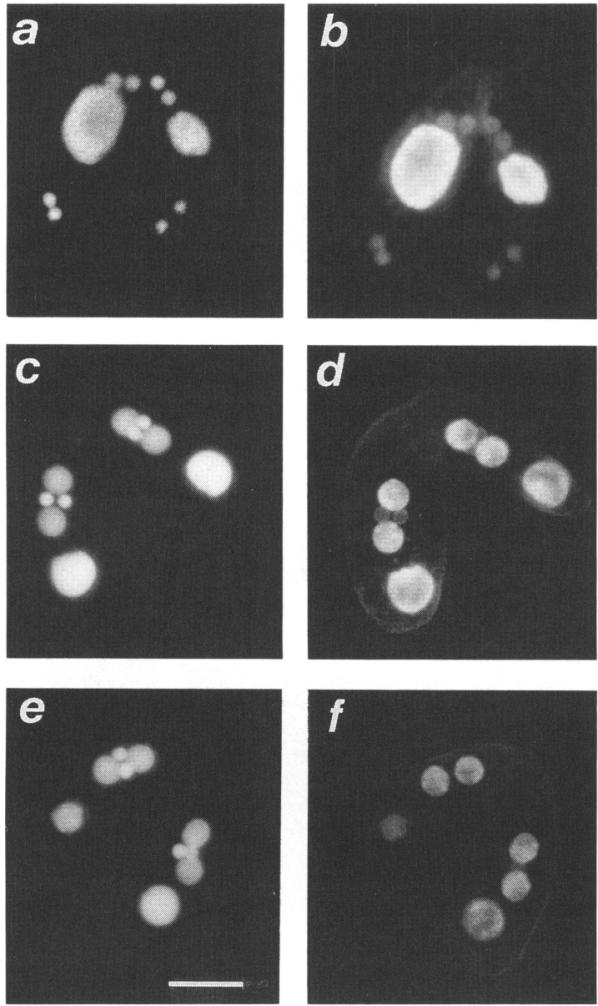

Figure 4. Later stages of conjugation show loss of anti-hvlHPLC staining from micronuclei. Immunofluorescent images of mating pairs were stained with DAPI $(a, c, e)$ or anti-hvlHPLC $(b, d, f) .(a, b)$ Early macronuclear development showing developing macronuclei in the anterior end of the cells and micronuclei in the posterior end, $\sim 7 \mathrm{hr}$ after mixing; $(c, d)$ macronuclear development II, micronuclei and developing macronuclei have migrated to the middle of the cell while the parental macronucleus has moved to the posterior, at $\sim 8 \mathrm{hr} ;\langle e, f\rangle$ late macronuclear development II showing anti-hvl-HPLC staining is lost from micronuclei and diminishes in the parental macronucleus, which is beginning to degrade, at $\sim 8.5 \mathrm{hr}$. Bar, $10 \mu \mathrm{m}$.

unit gravity, which separates the nuclei primarily on the basis of size (Wang and Allis 1993). The samples were adjusted to produce approximately equivalent histone loads and analyzed by SDS-PAGE followed by immunoblotting with anti-hvl-HPLC. As expected, macronuclei from all three stages contained significant amounts of hvl (Fig. 5a). Micronuclei from growing or starved cells contained trace amounts of hvl, probably attributable to small amounts of contaminating macronuclei. However, micronuclei isolated from conjugating cells clearly possessed a significant amount of hvl. The amount of hvl in micronuclei from early conjugants cannot be explained by extensive macronuclear contamination, as staining of the same blot with antibodies against the macronuclearspecific linker histone Hl (Chicoine et al. 1985) showed little or no staining of any of the micronuclear preparations (Fig. 5b). Final staining of this blot with anti-H4 serum demonstrated nearly equivalent loads for all samples (data not shown).
Estimates of the abundance of hvl in conjugating micronuclei were made by comparing the Ponceau $S$ stained with the immunostained blot. We estimate that micronuclei from early stage conjugants contain onefourth to one-third the amount of hv1 as growing cell macronuclei. Because $\sim 15 \%$ of the total $\mathrm{H} 2 \mathrm{~A}$ in growing cell macronuclei is hvl (Allis et al. 1980b), $\sim 5 \%$ of the $\mathrm{H} 2 \mathrm{~A}$ in micronuclei of early conjugants is hv1. Depending on whether nucleosomes contain two molecules of hvl or one molecule of hvl and one of either of the two major $\mathrm{H} 2 \mathrm{As}, 5-10 \%$ of the micronuclear nucleosomes contain hvl at this stage. Although these estimates are approximate, it seems clear that hvl is associated with more than a few micronuclear DNA sequences but not with the majority of the micronuclear genome.

These results confirm the developmentally regulated acquisition of hvl, initially observed via immunocytochemical techniques. They also demonstrate that not all macronuclear proteins accumulate in the micronuclei at this stage of development because $\mathrm{Hl}$ does not.

\section{Staining of conjugating cells with anti-peptide antisera}

Staining of conjugating pairs with anti-amino serum parallels the staining observed with the anti-hvl-HPLC serum. This serum stains parental macronuclei throughout development (Fig. 6). In addition, this serum stains meiotic (Fig. 6b) and gametic micronuclei (data not shown), as well as postzygotic nuclei (Fig. $6 \mathrm{~d}, \mathrm{f})$. Staining is restricted to macronuclei late during development (Fig. 6h).

Although anti-carboxy serum stains the parental ma-

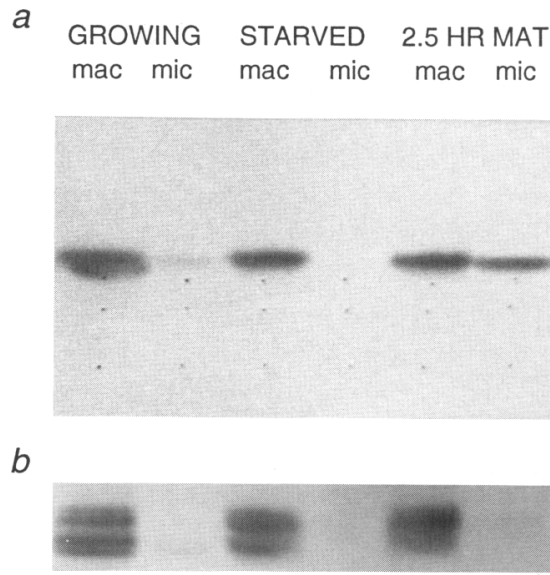

Figure 5. Biochemical purification of fixed nuclei and immunoblot analyses demonstrates the sudden acquisition of hvl in micronuclei during early conjugation. Nuclei were isolated from growing, starved, and conjugating ( $2.5 \mathrm{hr}$ postmixing) cultures and fractionated. (a) Macronuclear (mac) and micronuclear (mic) samples were separated on a SDS- $12 \%$ polyacrylamide gel and electroblotted to nitrocellulose. hvl was detected after incubation with anti-hvl-HPLC serum. (b) Subsequently, incubation with anti-Hl serum was used to assay for macronuclear contamination. Multiple bands represent previously described phosphorylated forms of $\mathrm{Hl}$ (Roth et al. 1988). 
Figure 6. Micronuclei are stained by antiamino antiserum but not by anti-carboxy antiserum in earlier stages conjugation; but at later stages of conjugation both antisera stain micronuclei. Immunofluorescent images of mating pairs stained with DAPI $\{a, c, e, g, i, k, m, o\}$, anti-amino serum $(b, d, f, h)$, or anti-carboxy serum $(j, 1, n, p)$. Micronuclei at meiotic prophase just beginning crescent formation show staining of micronuclei by the anti-amino antiserum $(b)$ but not by anti-carboxy antiserum (j). At macronuclear development I the anti-amino serum stains both the macronuclei and the micronuclei, which are positioned in the posterior of the cell $(d)$, but the anti-carboxy serum only stains the anterior developing macronuclei (1). At macronuclear development II anti-amino $(f)$ and anti-carboxy $(n)$ sera stain both micronuclei and developing macronuclei. At later macronuclear development II, when the parental macronuclei begin to degrade and lose immunostaining, neither antiserum stains micronuclei significantly $\{h, p)$. Bar, $10 \mu \mathrm{m}$.
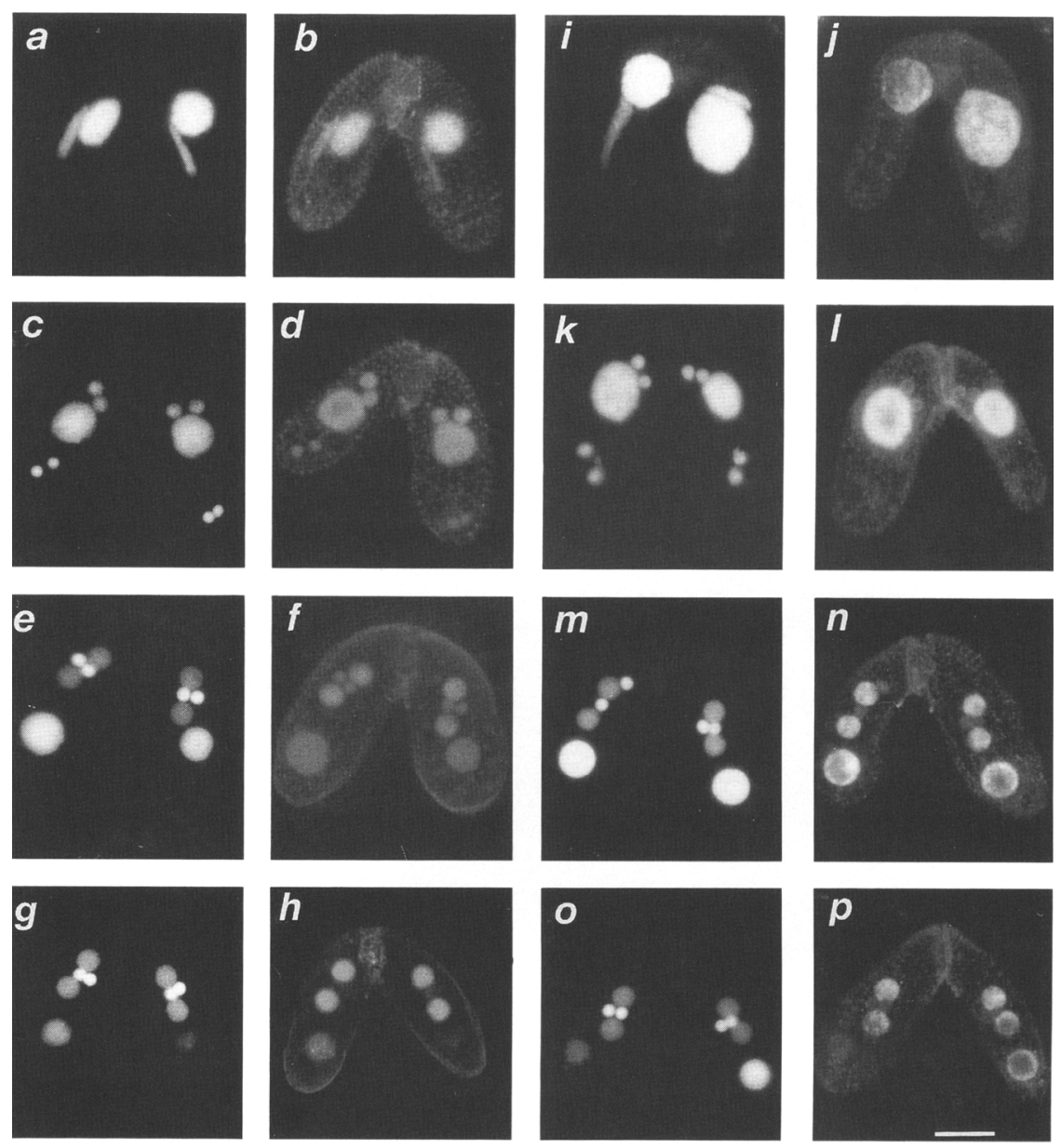

cronucleus throughout development, it does not detect hvl in micronuclei during the early or middle stages of conjugation (Fig. 6j,1), suggesting that this epitope is not accessible for interaction with the antibodies. However, anti-carboxy serum does stain micronuclei briefly (Fig. 6n), late in development, just before the loss of hvl detection by this (Fig. 6p) and the other two antisera.

\section{Discussion}

Eukaryotic DNA is packaged into nucleosomes via interaction with the four core histones, $\mathrm{H} 2 \mathrm{~A}, \mathrm{H} 2 \mathrm{~B}, \mathrm{H} 3$, and $\mathrm{H} 4$ (for review, see Isenberg 1979). Microheterogeneity of nucleosomes, either by secondary modifications of the histones or the incorporation of histone variants and/or nonhistone proteins, could provide a means for regulating the structural and functional state of the chromatin. Although the existence of histone variants is well established (Harvey and Whiting 1983; Grunstein et al. 1984; Zweidler 1984; Hatch and Bonner 1990; Waterborg 1991), the actual roles that these proteins play in the modulation of chromatin are not known. In an effort to gain insights into the functional significance of a histone variant, we have used immunocytochemistry to examine the nuclear distribution of hvl, an $\mathrm{H} 2 \mathrm{~A}$ variant, in
Tetrahymena. Because this variant is conserved throughout evolution, it is likely to play a fundamental role in chromatin structure and/or function.

We used synthetic peptides to the amino and carboxyl termini, as well as HPLC-purified whole protein, to generate three polyclonal antisera to hvl. Histones are comprised of three domains: an extended hydrophilic aminoterminal tail, a globular, hydrophobic core, and a hydrophilic carboxy-terminal tail (for review, see McGhee and Felsenfeld 1980). The core domain is involved largely in histone-histone interactions necessary for nucleosome stability, whereas the amino terminus is likely to interact with DNA (Hill and Thomas 1990) or with extranucleosomal proteins (Johnson et al. 1990) and serves as a site for acetylation (Bonner and Stedman 1979). The carboxy-terminal tail of histone $\mathrm{H} 2 \mathrm{~A}$ can be cross-linked to histone Hl (Bonner and Stedman 1979), and the digestion of this domain leads to decondensation of nucleosomal fibers (Hacques et al. 1990). Thus, this domain may be important for higher order chromatin structure because the presence of $\mathrm{H} 1$ in vitro can transform $10-\mathrm{nm}$ nucleosomal fibers into $30-\mathrm{nm}$ filaments (Van Holde 19891 .

Immunoblotting analyses were performed to determine the specificity of the three antisera. All three were 
specific for hvl under the conditions employed. Competition experiments using synthetic peptides established that anti-amino and anti-carboxy peptide antisera are specific for their immunogens. Anti-hv1-HPLC detection of hvl was only partially competed with carboxyterminal and variant box peptides and not detectably with amino-terminal peptide. This indicates that antihvl-HPLC serum recognizes several regions of hvl.

Immunofluorescence analyses demonstrated that the transcriptionally active macronuclei stained at all stages of the life cycle with all anti-hvl antisera. The transcriptionally inert micronuclei did not stain during growth or starvation but stained with anti-hv1-HPLC and antiamino sera during early stages of conjugation. The onset of micronuclear staining was $\sim 1-1.5 \mathrm{hr}$ after mixing cells, when the micronucleus migrates from its pocketlike recess in the macronuclear surface, where it usually resides in growing and starved cells. Staining by antiacetylated histone antibodies demonstrated that at least some of the hvl present at this stage as well as at later stages is acetylated (data not shown). Acetylation of histone tails has been associated with transcription (Perry and Annunziato 1989; Ruiz and Wahl 1990) and has the potential to destabilize nucleosomes and may facilitate access of regulatory molecules (Oliva et al. 1990; Lee et al. 1993). The micronuclei at this stage swell slightly and the punctate, highly condensed chromatin observed in nonconjugating cells is transformed into a dense homogenous core surrounded by a "halo" of less condensed chromatin (Wolfe et al. 1976), consistent with a change in chromatin structure attributable to the acquisition of hvl. The micronuclei then begin an extended period of elongation, termed the crescent stage, where partially condensed chromatin is organized into long, thin strands. The micronucleus is transcriptionally active during early crescent stage (the stage shown in Fig. 3c); this activity ceases by late crescent (Fig. 3e; Sugai and Hiwatashi 1974; Martindale et al. 1985). The detection of hvl precedes the period of micronuclear transcriptional activity and outlasts it as well. This suggests that hvl is required for the formation of active chromatin, rather than appearing secondarily as a consequence of transcriptional activity.

The hypothesis that hvl in micronuclei is associated with transcriptional activation is supported by observations using antibodies specific to TBP. TBP is a general factor required for transcription by all three nuclear RNA polymerases (for review, see White and Jackson 1992; Rigby 1993). Anti-TBP serum stains only macronuclei during growth and starvation but stains nuclei during conjugation with a pattern similar to that observed for anti-hvl; parental macronuclei, meiotic micronuclei, gametic and postzygotic nuclei stain with anti-TBP antibody (Stargell and Gorovsky 1993). AntiTBP staining disappears from micronuclei and is restricted to developing macronuclei late in development. Because TBP is a necessary component for transcriptional activation, it seems likely that the coincident appearance of hvl in conjugating micronuclei is associated with the transcriptional activation of these nuclei.
If hvl is solely involved in the transcription of micronuclear DNA, then why does it persist after transcription is no longer detectable? One possibility is that hvl may be required for micronuclear DNA replication, which also occurs during conjugation (Allis et al. 1987). However, because replicating micronuclei of growing cells do not stain detectably with any of the anti-hvl sera (data not shown), a function for hvl in replication seems unlikely. Another possibility is that hvl is required for the transcriptional activation of micronuclei early in conjugation and is simply not removed until late in development, after nuclear fates are determined. It may be of no consequence to the cell that this protein /and others) remains present during later stages of conjugation. However, it seems more likely that persistence of hvl in micronuclei after transcription ceases is a reflection of the fact that micronuclear chromatin remains in a transcriptionally competent state until late in conjugation. Nanney (1953) has shown that after the second postzygotic division, presumptive micronuclei, located in the posterior of the cell, can still differentiate into macronuclei if relocated anteriorly (see below).

Anti-hvl-HPLC and anti-amino sera staining disappeared from developing micronuclei late in conjugation. Interestingly, anti-carboxy antibodies stained micronuclei only briefly, just before this late stage of development. It may be that like the major H2As that have been shown to interact with H1 (Bonner and Stedman 1979), the carboxy-terminal tail of hvl in micronuclei interacts with a chromatin protein, thus obscuring this epitope from detection in situ. This hypothetical protein is not $\mathrm{H1}$, as there is no detectable immunofluorescent staining of $\mathrm{Hl}$ in these micronuclei (data not shown). The micronuclear linker histones are likely candidates, but it is not yet known whether they are present at this stage. The detection of the previously sequestered carboxy-terminal tail of hvl during late stages of conjugation may be related to the protein's subsequent removal, as it occurs just before the loss of all hvl staining in these maturing micronuclei. An anti-hvl-gel serum described earlier (Allis et al. 1982) produced the same pattern of staining as the anti-carboxy antibodies, in which only macronuclei and some micronuclei of late-stage conjugants were stained (Wenkert and Allis 1984). This is consistent with competition experiments demonstrating that this antiserum had characteristics indistinguishable from those of the anti-carboxy serum (data not shown). The changes in accessibility revealed by these experiments suggest that the conformation of hvl in the nucleosome or its association with other proteins may be altered.

The mechanism by which hv1 is eliminated from the micronucleus appears to be an active process, as micronuclei do not enlarge or divide at this time. During this transition period hvl is not the only protein to be eliminated from micronuclei. Antibodies specific to TBP /described above) and high mobility group protein B (HMG B) also cease to stain micronuclei at this transition stage (Wang and Allis 1993). Thus, the developing micronuclei appear to be undergoing a dynamic chromatin reorganization at this transition period, which may be linked to 
the restriction of transcriptional and developmental potential in these nuclei, and must eventually include the acquisition of the micronuclear-specific linker histones. The developmental signals that trigger this chromatin transition are unknown. Although this restructuring occurs when the developing macronuclei and micronuclei are in close proximity in the cytoplasm, the fates of these nuclei are actually determined much earlier, after the second postzygotic division, when the products of this division are oriented such that two nuclei are located in the anterior and two in the posterior of the cell (as shown in Fig. 4a). Nanney's results (Nanney 1953) strongly support a determinative role of localized cytoplasmic regions, as nuclei relocated to the anterior region by centrifugation develop into macronuclei, whereas those relocated to the posterior region become micronuclei. At later stages developing macro- and micronuclei come together near the center of the cell, and position is no longer important. Thus, nuclear determinants must be partitioned to the anterior and/or posterior nuclei, which thereby initiate the appropriate developmental process. One of the events triggered in the micronucleus is the elimination of proteins destined to be macronucleus specific in vegetative cells. After the transition period, there must either be selective deposition of these proteins into macronuclei or selective degradation of them in micronuclei to maintain the macronuclear-specific localization observed in growing and starved cells. Although micronuclei in the later stages of conjugation are no longer pluripotent and the distinct function and compositions of macro- and micronuclei can be maintained and replicated through hundreds of vegetative divisions, this restriction of nuclear fate can be developmentally reversed. The micronuclei are responsible for the genetic continuity of the organism and, during the next cycle of conjugation, must ultimately give rise to the subsequent generation of macro- and micronuclei.

Both the parental macronucleus and the developing macronuclei are immune to the events that remodel chromatin in the micronuclei. Each is experiencing its own unique alterations in chromatin structure during this transition period. The parental macronucleus undergoes programmed nuclear death, a process characterized by progressive reduction in size of DNA, proteolysis of histones, and transformation of the chromatin into a highly condensed state (Davis et al. 1992). As noted previously (Orias 1986), similar events occur in nuclei of animal cells undergoing apoptosis. In contrast, the developing macronuclei continue to mature and differentiate. Macronuclear differentiation is characterized by accumulation of $\mathrm{Hl}$, large-scale acetylation of histones (Lin et al. 1989), chromosome fragmentation, DNA sequence elimination, rearrangement and amplification, and the onset of gene expression (for review, see Gorovsky 1980; Blackburn and Karrer 1986; Brunk 1986; Yao 1989).

The studies presented here argue that there is a tight correlation between the presence of the histone variant hvl and transcriptionally competent chromatin. With the development of techniques for gene replacement
(Yao and Yao 1991; Kahn et al. 1993) and mass transformation of Tetrahymena (Gaertig and Gorovsky 1992), additional studies aimed directly at determining the in vivo function of this histone variant should now be possible.

\section{Materials and methods}

Cells and culture conditions

T. thermophila (strains B2086, mating type II, and CU428, mating type VII; kindly provided by P.J. Bruns, Cornell University, Ithaca, NY) were grown axenically in enriched proteose peptone at $30^{\circ} \mathrm{C}$ as described (Gorovsky et al. 1975). Cells were starved at a density of $2 \times 10^{5}$ to $3 \times 10^{5}$ cells $/ \mathrm{ml}$ in $10 \mathrm{mM}$ Tris $-\mathrm{HCl}$ at $\mathrm{pH}$ 7.4 for $18-22 \mathrm{hr}$, without shaking. Conjugation was induced by mixing equal numbers of cells of different mating types.

\section{Peptide antisera preparation}

Peptides corresponding to three regions of the $T$. thermophila hvl protein were prepared by solid-phase peptide synthesis on an Applied Biosystems model 430A peptide synthesizer and subjected to amino acid analysis with a Waters Associates Pico Tag System (Fig. 1a): amino peptide, VGGAKNKKTPQSGGC; variant box peptide, GRVSAKNRVGATGGC; and carboxy peptide, CGKHSTKNRSSAKTAEPR. The underlined amino acids are not part of the protein but serve as spacers (glycines) or as a coupler (cysteine) between the peptide and the carrier protein, keyhole limpet hemocyanin (KLH, Sigma). Coupling was accomplished by a modification of described methods (Lerner et al. 1981; Doolittle 1986). Hemocyanin (20 mg) was briefly sonicated into a suspension in $0.25 \mathrm{ml}$ of $50 \mathrm{~mm}$ phosphate, $100 \mathrm{~mm}$ $\mathrm{NaCl}$ at pH 6.5, with a Branson Sonifier model 200 and microprobe tip. Ten microliters of $m$-maleimidobenzoyl- $N$-hydroxysuccinimide ester (MBS; Pierce) at $150 \mathrm{mg} / \mathrm{ml}$ in dimethylformamide was added with vortexing, and the reaction allowed to proceed for $30 \mathrm{~min}$ at room temperature with agitation. Free MBS was removed by chromatography on a Sephadex G-25 column (Pharmacia) and the protein-containing fractions $\left(A_{280}\right)$ were pooled and the $\mathrm{pH}$ adjusted to 7.5 . Approximately $3-5 \mathrm{mg}$ of peptide in $0.1-0.2 \mathrm{ml}$ of phosphate-buffered saline (PBS; 150 $\mathrm{mM} \mathrm{NaCl}, 10 \mathrm{~mm}$ sodium phosphate at $\mathrm{pH} 7.4$ ) was added, and the coupling reaction was allowed to proceed for 3-4 hr. Coupling efficiency (25-50 molecules of peptide per $10^{5}$ daltons of $\mathrm{KLH}$ ) was determined by quantitation of unreacted sulfhydryl groups by the method of Stadtman (1957).

Immunization of New Zealand white rabbits (Dutchland) with peptide-KLH conjugants was performed by emulsifying 1 mg suspension of the peptide-KLH complex in water with an equal volume of complete Freund's adjuvant (Sigma) and injecting this subcutaneously. Secondary immunizations were performed 2 weeks later with the peptide-KLH complex in incomplete Freund's adjuvant. Blood was obtained from an ear vein weekly starting 1 week after the secondary injection. Preimmune serum was obtained before immunization. Serum was obtained by centrifugation $\left(2200 \mathrm{~g}\right.$ at $4^{\circ} \mathrm{C}$ ) of clotted blood (several hours at $4^{\circ} \mathrm{C}$ ) and was stored at $-70^{\circ} \mathrm{C}$.

\section{Histone purification}

Histones were extracted from Tetrahymena macronuclei with $0.4 \mathrm{~N} \mathrm{H}_{2} \mathrm{SO}_{4}$ (Allis et al. 1979) and precipitated with $0.5 \mathrm{~N}$ perchloric acid. The acid-insoluble pellet was dissolved in $4 \mathrm{~m}$ urea, $5 \% \beta$-mercaptoethanol, and centrifuged for $5 \mathrm{~min}$ at $16,000 \mathrm{~g}$ to 
remove particulates. Two consecutive rounds of reverse-phase HPLC were performed using a C8 analytical column (Aquapore RP-300, Applied Biosystems, Santa Clara, CA). The first round employed a linear gradient of $0-60 \%$ buffer B in A over $30 \mathrm{~min}$, where buffer $\mathrm{A}$ was $5 \%$ acetonitrile, $0.1 \%$ trifluoroacetic acid (TFA) in water and buffer B was $90 \%$ acetonitrile, $0.1 \%$ TFA in water. Fractions were eluted at $1 \mathrm{ml} / \mathrm{min}$, monitored at $214 \mathrm{~nm}$, and subjected to a second round of purification using a gradient of $0-40 \% \mathrm{~B}$ in A over $15 \mathrm{~min}$ followed by $40-65 \% \mathrm{~B}$ over 30 min. Final histone fractions were dried under vacuum.

\section{Histone antisera generation}

hvl antibodies were obtained from rabbit immunizations using the services of the Pocono Rabbit Farm and Laboratory (Canadensis, Pennsylvania). Preimmune sera from four rabbits were screened for low immunoprecipitation of chromatin (Dedon et al. 1991) and background staining on immunoblots of Tetrahymena macronuclear proteins. Additional preimmune serum was obtained from the rabbit with lowest background and that rabbit was subjected to the following immunization schedule: Subcutaneous injection of Freund's adjuvant was followed 1 week later by intradermal injection of $30 \mu \mathrm{g}$ of the HPLC-purified hvl protein in Freund's complete adjuvant; this injection was repeated 2 weeks later. Bleeds were taken weekly, and an intraperitoneal boost of $3 \mu \mathrm{g}$ of hvl protein was given 8 weeks after injections began. Sera obtained were aliquoted and stored at $-70^{\circ} \mathrm{C}$.

Polyclonal rabbit anti-H2A and anti- $\mathrm{H} 4$ sera were generated from fractions of HPLC-purified samples at Syracuse University with the following injection schedule: An intradermal injection of $30 \mu \mathrm{g}$ of the purified histone samples in Freund's complete adjuvant was given, and this treatment was repeated 2 weeks later using Freund's incomplete adjuvant. Bleeds were taken weekly. Serum was obtained by centrifugation $\left(2200 \mathrm{~g}\right.$ at $\left.4^{\circ} \mathrm{C}\right)$ of clotted blood (several hours at $4^{\circ} \mathrm{C}$ ) and was stored at $-70^{\circ} \mathrm{C}$.

\section{Gel electrophoresis}

Tetrahymena histones were extracted and separated on SDS- or triton/acid/urea-polyacrylamide gels as described previously (Allis et al. 1979, 1980a). Samples for electroblotting were run on wide preparative gels.

\section{Immunoblotting and peptide competition}

Standard immunoblotting procedures were used (Towbin et al. 1979; Harlow and Lane 1988). SDS gels were blotted to nitrocellulose using a semidry electrophoretic transfer unit (Biotrans, Gelman, Ann Arbor, MI) with $48 \mathrm{~mm}$ Tris-Base, $39 \mathrm{~mm}$ glycine, $0.1 \%$ SDS, and $20 \%$ methanol buffer. To determine transfer efficiency, blots were stained with $0.02 \%$ Ponceau $S$ in $0.3 \%$ trichloroacetic acid and destained in water. All incubations with sera and blocking were performed in blocking solution containing $5.0 \%$ (wt/vol) nonfat dry milk, $0.01 \%$ antifoam A (Sigma), $0.0001 \%$ merthiolate; in $1 \times \mathrm{TBS}(150 \mathrm{mM} \mathrm{NaCl}$ and $50 \mathrm{~mm}$ Tris- $\mathrm{HCl}$ at $\mathrm{pH} 8.1) . \mathrm{NaCl}(300 \mathrm{~mm})$ was added to the primary antibody reaction and first TBS wash to reduce nonspecific binding. A small amount of cross-reactivity to other histones was seen if the antisera were too concentrated or $\mathrm{NaCl}$ was omitted. Primary antibodies were used at 1:1000 dilution except for anti-hv1-HPLC, which was used at 1:10,000. Incubation was overnight at $4^{\circ} \mathrm{C}$. Following reaction with peroxidase-conjugated goat anti-rabbit antiserum (Sigma, 1:2000 dilution), the blots were developed with HRP Color Development Reagent (Bio-Rad) according to the manufacturer's instructions.
Immunoblot competition was performed using 100-fold (for anti-peptide antisera) or 1000-fold (for anti-hvl-HPLC antiserum) molar excess of peptide to antibody. This ratio was calculated using an estimate of $10 \mathrm{mg}$ of $\mathrm{IgG} / \mathrm{ml}$ of antiserum (Harlow and Lane 1988) without determination of specific hv1 IgG concentration; therefore, fold excesses given are minimum values. Peptide and serum were combined in $100 \mu \mathrm{l}$ of blocking solution and incubated for $3 \mathrm{hr}$ at $30^{\circ} \mathrm{C}$. This reaction mixture was diluted in blocking solution and used for primary antibody incubation as described above.

\section{Biochemical analyses of separated macro- and micronuclei}

Purification of macro- and micronuclei by sedimentation at unit gravity, FACScan analyses, gel electrophoresis, and immunoblotting were performed as described (Wang and Allis 1993). Western blot antisera incubations were performed successively with anti-hvl-HPLC serum (diluted 1:18,000), anti-Hl serum (diluted 1:300, Chicoine et al. 1985), and anti-H4 serum (diluted $1: 2000)$.

\section{Cell fixation}

Cells were extracted and fixed as described /Gaertig and Fleury 1992) with the following modifications. Cells were incubated for $3 \mathrm{~min}$ at room temperature in $0.5 \%$ Triton X-100 in PHEM buffer, washed, and then fixed in freshly prepared $1 \%$ paraformaldehyde in PBS. Fixed cells were washed in PBS and stored at $4^{\circ} \mathrm{C}$ for up to 1 week, and $50 \mu$ l of cell suspension was then applied to poly-L-lysine (Sigma)-coated coverslips and allowed to dry.

\section{Indirect immunofluorescent staining and microscopy}

To reduce nonspecific sticking, coverslips with dried cells were first incubated for $1 \mathrm{hr}$ at $37^{\circ} \mathrm{C}$ in blocking solution: $10 \%$ normal goat serum (GIBCO), 3\% BSA, $0.2 \%$ Tween 20 (Sigma), in PBS. Coverslips were incubated with primary antiserum overnight at $4^{\circ} \mathrm{C}$. Primary antisera were diluted in blocking solution at the following concentrations: anti-hvl-HPLC (immune and preimmune), 1:500; anti-amino serum, 1:100; anti-carboxy serum, 1:100; and anti-H2A serum, 1:500. Coverslips were washed three times in PBS-0.2\% Tween 20 and treated for $2 \mathrm{hr}$ with secondary antibody, FITC-conjugated goat anti-rabbit antiserum (Sigma) diluted 1:160 in PBS-0.2\% Tween 20. After washing once with PBS, cells were stained with the DNAspecific dye 4',6-diamidine-2-phenyl-indole dihydrochloride (DAPI, Boehringer Mannheim) at $5 \mathrm{ng} / \mathrm{ml}$ in PBS for $5 \mathrm{~min}$, followed by a PBS wash. Cells were photographed using an Olympus BH-2 microscope equipped for fluorescence and filters specific for fluorescein to visualize the antisera staining or DAPI to show the position of the nuclei.

\section{Acknowledgments}

This work was supported by grants to M.A.G. from the American Cancer Society (NP-717A) and the National Institutes of Health (NIH; GM21793) and to C.D.A. from the NIH (HD16259). P.C.D was supported by a James P. Wilmot Cancer Research Fellowship. Special thanks go to T.H. Thatcher for preparation of histone samples and for critical reading of this manuscript.

The publication costs of this article were defrayed in part by payment of page charges. This article must therefore be hereby marked "advertisement" in accordance with 18 USC section 1734 solely to indicate this fact. 


\section{References}

Allis, C.D., C.D.C. Glover, and M.A. Gorovsky. 1979. Micronuclei of Tetrahymena contain two types of histones H3. Proc. Natl. Acad. Sci. 76: 4857-4861.

Allis, C.D., J.K. Bowen, G.N. Abraham, C.V.C. Glover, and M.A. Gorovsky. 1980a. Proteolytic processing of histone H3 in chromatin. A physiologically regulated event in Tetrahy. mena micronuclei. Cell 20: 55-64.

Allis, C.D., C.V.C. Glover, J.K. Bowen, and M.A. Gorovsky. $1980 \mathrm{~b}$. Histone variants specific to the transcriptionally active, amitotically dividing macronucleus of the unicellular eukaryote, Tetrahymena thermophila. Cell 20: 609-617.

Allis, C.D., Y.S. Ziegler, M.A. Gorovsky, and J.B. Olmsted. 1982. A conserved histone variant enriched in nucleoli of mammalian cells. Cell 31: 131-136.

Allis, C.D., R.L. Allen, J.C. Wiggins, L.C. Chicoine, and R. Richman. 1984. Proteolytic processing of H1-like histones in chromatin: A physiologically and developmentally regulated event in Tetrahymena micronuclei. J. Cell Biol. 99: 1669_ 1677.

Allis, C.D., R. Richman, M.A. Gorovsky, Y.S. Ziegler, B. Touchstone, W.A. Bradley, and R.G. Cook. 1986. hvl is an evolutionarily conserved $\mathrm{H} 2 \mathrm{~A}$ variant that is preferentially associated with active genes. J. Biol. Chem. 261: 1941-1948.

Allis, C.D., M. Colavito-Shepanski, and M.A. Gorovsky. 1987. Scheduled and unscheduled DNA synthesis during development in conjugating Tetrahymena. Dev. Biol. 124: 469-480.

Blackburn, E.H. and K.M. Karrer. 1986. Genomic reorganization in ciliated protozoans. Annu. Rev. Genet. 20: 501-521.

Bonner, W.M. and J.D. Stedman. 1979. Histone 1 is proximal to histone 2A and to $\mathrm{A}_{24}$. Proc. Natl. Acad. Sci. 76: 2190-2194.

Brunk, C.F. 1986. Genome reorganization in Tetrahymena. Int. Rev. Cytol. 99: 49-83.

Chicoine, L.G., D. Wenkert, R. Richman, J.C. Wiggins, and C.D. Allis. 1985. Modulation of linker histones during development in Tetrahymena: Selective elimination of linker histone during the differentiation of new macronuclei. Dev. Biol. 109: 1-8.

Chicoine, L.G., R. Richman, R.C. Cook, M.A. Gorovsky, and C.D. Allis. 1987. A single histone acetyltransferase from Tetrahymena macronuclei catalyzes deposition-related acetylation of free histones and transcription-related acetylation of nucleosomal histones. I. Cell Biol. 105: 127-135.

Dalton, S., A.J. Robins, R.P. Harvey, and J.R.E. Wells. 1989. Transcription from the intron-containing chicken histone

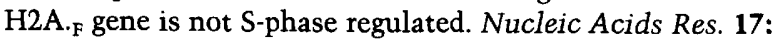
$1745-1756$.

Davis, M.C., J.G. Ward, G. Herrick, and C.D. Allis. 1992. Programmed nuclear death: Apoptotic-like degradation of specific nuclei in conjugating Tetrahymena. Dev. Biol. 154: 419-432.

Dedon, P.C., J.A. Soults, C.D. Allis, and M.A. Gorovsky. 1991. A simplified formaldehyde fixation and immunoprecipitation technique for studying protein-DNA interactions. Anal. Biochem. 197: 83-90.

Doolittle, R. 1986. Of URFs and ORFs. A primer on how to analyze derived amino acid sequences. University Science Books, Mill Valley, CA.

Ernst, S.G., H. Miller, C.A. Brenner, C. Nocente-McGrath, S. Francis, and R. McIsaac. 1987. Characterization of a cDNA clone coding for a sea urchin histone $\mathrm{H} 2 \mathrm{~A}$ variant related to the H2A.F/Z histone protein in vertebrates. Nucleic Acids Res. 15: 4629-4644.

Gaertig, J. and A. Fleury. 1992. Spatio-temporal reorganization of intracytoplasmic microtubules is associated with nuclear selection and differentiation during the developmental process in the ciliate Tetrahymena thermophila. Protoplasma 167: 74-87.

Gaertig, J. and M.A. Gorovsky. 1992. Efficient mass transformation of Tetrahymena thermophila by electroporation of conjugants. Proc. Natl. Acad. Sci. 89: 9196-9200.

Gorovsky, M.A. 1973. Macro- and micronuclei of Tetrahymena pyriformis: A model system for studying the structure and function of eukaryotic nuclei. $J$. Protozool. 20: 19-25.

. 1980. Genome organization and reorganization in Tetrahymena. Annu. Rev. Genet. 14: 203-239.

1986. Ciliate chromatin and histones. In The Molecular Biology of Ciliated Protozoa (ed. J.G. Gall), pp. 227-261. Academic Press Inc., Orlando.

Gorovsky, M.A., J.B. Keevert, and G.L. Pleger. 1974. Histone F1 of Tetrahymena macronuclei. Unique electrophoretic properties and phosphorylation of $\mathrm{Fl}$ in an amitotic nucleus. $J$. Cell Biol. 61: 134-145.

Gorovsky, M.A., M.-C. Yao, J.B. Keevert, and G.L. Pleger. 1975. Isolation of micro- and macronuclei of Tetrahymena pyriformis. In Methods in cell biology (ed. P.M. Prescott), pp. 311-327. Academic Press, New York.

Grunstein, M. 1990. Nucleosomes: Regulators of transcription. Trends Genet. 6: 395-400.

Grunstein, M., M. Rykowski, D. Kolodrubetz, J. Choe, and J. Wallis. 1984. A genetic analysis of histone protein subtypes in yeast. In Histone genes. (eds. G.S. Stein, J.L. Stein, and W.F. Marzluff), pp. 35-63. John Wiley \& Sons, New York.

Hacques, M.-F., S. Muller, G. De Murcia, M.H.V. Van Regenmortel, and C. Marion. 1990. Accessibility and structural role of histone domains in chromatin. Biophysical and immunochemical studies of progressive digestion with immobilized proteases. I. Biomol. Struct. \&) Dynam. 8: 619-641.

Harlow, E. and D. Lane. 1988. Antibodies: A laboratory manual Cold Spring Harbor Laboratory, Cold Spring Harbor, New York.

Harvey, R.P. and J.A. Whiting. 1983. H2A.F: An extremely variant histone $\mathrm{H} 2 \mathrm{~A}$ sequence expressed in the chicken embryo. Proc. Natl. Acad. Sci. 80: 2819-2823.

Hatch, C.L. and W.M. Bonner. 1988. Sequence of cDNAs for mammalian H2A.Z, an evolutionarily diverged but highly conserved basal histone H2A isoprotein species. Nucleic Acids Res. 16: 1113-1124.

1990. The human histone H2A.Z gene. Sequence and regulation. J. Biol. Chem. 265: 15211-15218.

Hill, C.S. and J.O. Thomas. 1990. Core histone-DNA interactions in sea urchin sperm chromatin-The N-terminal tail of H2B interacts with linker DNA. Eur. J. Biochem. 187: 145153.

Isenberg, I. 1979. Histones. Annu. Rev. Biochem. 48: 159-191.

Johmann, C.A. and M.A. Gorovsky. 1976. Immunofluorescence evidence for the absence of histone $\mathrm{H} 1$ in a mitotically dividing, genetically inactive nucleus. $/$. Cell Biol. 71: 89-95.

Johnson, L.M., P.S. Kayne, E.S. Kahn, and M. Grunstein. 1990. Genetic evidence for an interaction between SIR3 and histone $\mathrm{H} 4$ in the repression of the silent mating loci in Saccharomyces cerevisiae. Proc. Natl. Acad. Sci. 87: 62866290.

Kahn, R.W., B.H. Andersen, and C.F. Brunk. 1993. Transformation of Tetrahymena thermophila by the microinjection of a foreign gene. Proc. Natl. Acad. Sci. 90: 9295-9299.

Lee, D.Y., J.J. Hayes, D. Pruss, and A.P. Wolffe. 1993. A positive role for histone acetylation in transcription factor access to nucleosomal DNA. Cell 72: 73-84.

Lerner, R.A., N. Green, H. Alexander, F.T. Liu, J.G. Sutcliffe, and T.M. Shinnick. 1981. Chemically synthesized peptides 
predicted from the nucleotide sequence of the hepatitis B virus genome elicit antibodies reactive with the native envelope of Dane particles. Proc. Natl. Acad. Sci. 78: 34033407.

Lin, R., J.W. Leone, R.G. Cook, and C.D. Allis. 1989. Antibodies specific to acetylated histones document the existence of deposition- and transcription-related histone acetylation in Tetrahymena. J. Cell Biol. 108: 1577-1588.

Martindale, D.W., C.D. Allis, and P.J. Bruns. 1982. Conjugation in Tetrahymena thermophila. A temporal analysis of cytological stages. Exp. Cell Res. 140: 227-236.

- 1985. RNA and protein synthesis during meiotic prophase in Tetrahymena thermophila. J. Protozool. 32: 644-649.

McGhee, J.D. and G. Felsenfeld. 1980. Nucleosome structure. Annu. Rev. Biochem. 49: 1115-1156.

Nanney, D.L. 1953. Nucleo-cytoplasmic interaction during conjugation in Tetrahymena. Biol. Bull. 105: 133-148.

Oliva, R., D.P. Bazett-Jones, L. Locklear, and G.H. Dixon. 1990. Histone hyperacetylation can induce unfolding of the nucleosome core particle. Nucleic Acids Res. 18: 2739-2747.

Orias, E. 1986. Ciliate conjugation. In The molecular biology of ciliated protozoa (ed. J.G. Gall), pp. 45-84. Academic Press, Orlando, FL.

Park, E.-C. and J.W. Szostak. 1990. Point mutations in the yeast histone $\mathrm{H} 4$ gene prevent silencing of the silent mating type locus HML. Mol. Cell. Biol. 10: 4932-4934.

Perry, C.A. and A.T. Annunziato. 1989. Influence of histone acetylation on the solubility, $\mathrm{Hl}$ content and DNase I sensitivity of newly assembled chromatin. Nucleic Acids Res. 17: 4275-4291.

Rigby, P.W.J. 1993. Three in one and one in three: It all depends on TBP. Cell 72: 7-10.

Roth, S.Y., I.G. Schulman, R. Richman, R.G. Cook, and C.D. Allis. 1988. Characterization of phosphorylation sites in histone $\mathrm{Hl}$ in the amitotic macronucleus of Tetrahymena during different physiological states. J. Cell Biol. 107: 24732482.

Ruiz, J.C. and G.M. Wahl. 1990. Chromosomal destabilization during gene amplification. Mol. Cell. Biol. 10: 3056-3066.

Sadler, L.A. and C.F. Brunk. 1992. Phylogenetic relationships and unusual diversity in histone $\mathrm{H} 4$ proteins within the Tetrahymena pyriformis complex. Mol. Biol. Evol. 9: 70-84

Schümperli, D. 1986. Cell-cycle regulation of histone gene expression. Cell 45: 471-472.

Stadtman, E.R. 1957. Preparation and assay of acyl coenzyme A and other thiol esters; use of hydroxylamine. Methods Enzymol. 3: 931-941.

Stargell, L.A. and M.A. Gorovsky. 1993. TATA-binding protein and nuclear differentiation in Tetrahymena thermophila. Mol. and Cell. Biol. (in press).

Sugai, T. and K. Hiwatashi. 1974. Cytological and autoradiographic studies of the micronucleus at meiotic prophase in Tetrahymena pyriformis. J. Protozool. 21: 542-548.

Towbin, H., T. Staehelin, and J. Gordon. 1979. Electrophoretic transfer of proteins from polyacrylamide gels to nitrocellulose sheets: Procedure and some applications. Proc. Natl. Acad. Sci. 76: 4350-4354.

Van Daal, A. and S.C.R. Elgin. 1992. A histone variant, H2AvD, is essential in Drosophila melanogaster. Mol. Biol. Cell 3: 593-602.

Van Daal, A., E.M. White, M.A. Gorovsky, and S.C.R. Elgin. 1988. Drosophila has a single copy of the gene encoding a highly conserved histone $\mathrm{H} 2 \mathrm{~A}$ variant of the H2A.F/Z type. Nucleic Acids Res. 16: 7487-7498.

Van Daal, A., E.M. White, S.C.R. Elgin, and M.A. Gorovsky.
1990. Conservation of intron position indicates separation of major and variant $\mathrm{H} 2 \mathrm{As}$ is an early event in the evolution of eukaryotes. J. Mol. Evol. 30: 449-455.

Van Holde, K.E. 1989. Chromatin (Springer-Verlag, New York).

Vavra, K.J., C.D. Allis, and M.A. Gorovsky. 1982. Regulation of histone acetylation in Tetrahymena macro- and micronuclei. J. Biol. Chem. 257: 2591-2598.

Wang, T. and C.D. Allis. 1993. An abundant high-mobilitygroup-like protein is targeted to micronuclei in a cell cycledependent and developmentally regulated fashion in Tetrahymena thermophila. Mol. Cell. Biol. 13: 163-173.

Waterborg, J.H. 1991. Multiplicity of histone H3 variants in wheat, barley, rice, and maize. Plant Physiol. 96: 453-458.

Wenkert, D. and C.D. Allis. 1984. Timing of the appearance of macronuclear-specific histone variant hvl and gene expression in developing new macronuclei of Tetrahymena thermophila. J. Cell Biol. 98: 2107-2117.

White, E.M., D.L. Shapiro, C.D. Allis, and M.A. Gorovsky. 1988. Sequence and properties of the message encoding Tetrahymena hv1, a highly evolutionarily conserved histone H2A variant that is associated with active genes. Nucleic Acids Res. 16: 179-198.

White, R.J. and S.P. Jackson. 1992. The TATA-binding protein: A central role in transcription by RNA polymerases I, II and III. Trends Genet. 8: 284-288.

Wolfe, J., B. Hunter, and W.S. Adair. 1976. A cytological study of micronuclear elongation during conjugation in Tetrahymena. Chromosoma 55: 289-308.

Wu, M., C.D. Allis, and M.A. Gorovsky. 1988. Cell-cycle regulation as a mechanism for targeting proteins to specific DNA sequences in Tetrahymena thermophila. Proc. Natl. Acad. Sci. 85: 2205-2209.

Wu, R.S. and W.M. Bonner. 1981. Separation of basal histone synthesis from S-phase histone synthesis in dividing cells. Cell 27: 321-330.

Wu, R.S., S. Tsai, and W.M. Bonner. 1982. Patterns of histone variant synthesis can distinguish G0 from G1 cells. Cell 31: 367-374.

Yao, M.-C. 1989. Site-specific chromosome breakage and DNA deletion in ciliates. In Mobile DNA (ed. D. Berg and M. Howe), pp. 713-734. American Society for Microbiology, Washington, DC.

Yao, M.-C. and C.-H. Yao. 1991. Transformation of Tetrahymena to cycloheximide resistance with a ribosomal protein gene through sequence replacement. Proc. Natl. Acad. Sci. 88: 9493-9497.

Zweidler, A. 1984. Core histone variants of the mouse: Primary structure and differential expression. In Histone genes, (ed. G.S. Stein, J.L. Stein, and W.F. Marzluff), pp. 339-369. John Wiley \& Sons, New York.

. 1992. Role of individual histone tyrosines in the formation of the nucleosome complex. Biochemistry 31: 92059211. 


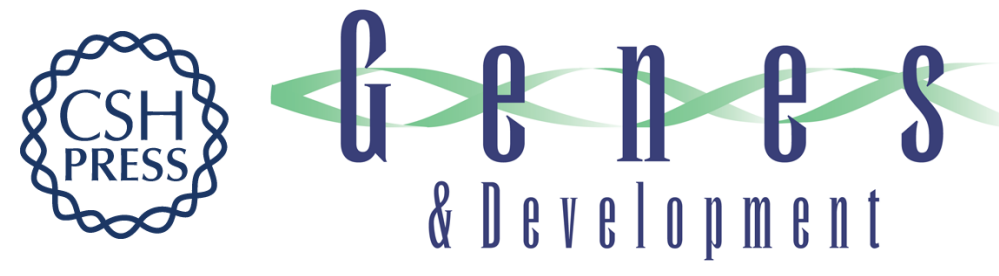

\section{Temporal and spatial association of histone H2A variant hv1 with transcriptionally competent chromatin during nuclear development in Tetrahymena thermophila.}

L A Stargell, J Bowen, C A Dadd, et al.

Genes Dev. 1993, 7:

Access the most recent version at doi:10.1101/gad.7.12b.2641

References This article cites 64 articles, 24 of which can be accessed free at:

http://genesdev.cshlp.org/content/7/12b/2641.full.html\#ref-list-1

License

Email Alerting

Service

Receive free email alerts when new articles cite this article - sign up in the box at the top right corner of the article or click here.

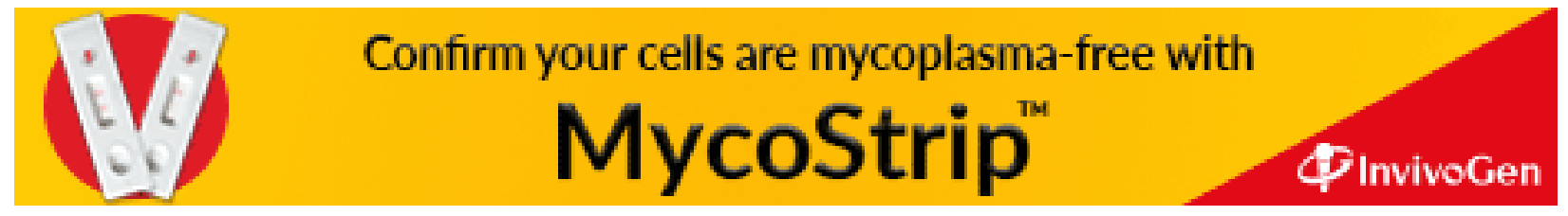

